Check for updates

Cite this: Chem. Sci., 2020, 11, 4312

๑ All publication charges for this article have been paid for by the Royal Society of Chemistry
Received 30th January 2020 Accepted 20th March 2020

DOI: $10.1039 / \mathrm{dOsc00569j}$

rsc.li/chemical-science

\section{SAXS studies of the thermally-induced fusion of diblock copolymer spheres: formation of hybrid nanoparticles of intermediate size and shape $\uparrow$}

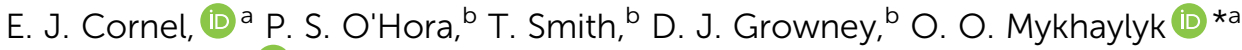 \\ and S. P. Armes (1D)*a
}

Dilute dispersions of poly(lauryl methacrylate)-poly(benzyl methacrylate) (PLMA-PBzMA) diblock copolymer spheres (a.k.a. micelles) of differing mean particle diameter were mixed and thermally annealed at $150{ }^{\circ} \mathrm{C}$ to produce spherical nanoparticles of intermediate size. The two initial dispersions were prepared via reversible addition-fragmentation chain transfer (RAFT) dispersion polymerization of benzyl methacrylate in $n$-dodecane at $90^{\circ} \mathrm{C}$. Systematic variation of the mean degree of polymerization of the core-forming PBzMA block enabled control over the mean particle diameter: small-angle $\mathrm{X}$-ray scattering (SAXS) analysis indicated that PLMA $39-\mathrm{PBzMA}_{97}$ and $\mathrm{PLMA}_{39}-\mathrm{PBzMA}_{294}$ formed well-defined, non-interacting spheres at $25^{\circ} \mathrm{C}$ with core diameters of $21 \pm 2 \mathrm{~nm}$ and $48 \pm 5 \mathrm{~nm}$, respectively. When heated separately, both types of nanoparticles regained their original dimensions during a $25-150-25^{\circ} \mathrm{C}$ thermal cycle. However, the cores of the smaller nanoparticles became appreciably solvated when annealed at $150{ }^{\circ} \mathrm{C}$, whereas the larger nanoparticles remained virtually non-solvated at this temperature. Moreover, heating caused a significant reduction in mean aggregation number for the $\mathrm{PLMA}_{39}-\mathrm{PBzMA}_{97}$ nanoparticles, suggesting their partial dissociation at $150{ }^{\circ} \mathrm{C}$. Binary mixtures of $\mathrm{PLMA}_{39}-\mathrm{PBzMA}_{97}$ and $\mathrm{PLMA}_{39}-\mathrm{PBzMA}_{294}$ nanoparticles were then studied over a wide range of compositions. For example, annealing a $1.0 \% \mathrm{w} / \mathrm{w}$ equivolume binary mixture led to the formation of a single population of spheres of intermediate mean diameter $(36 \pm 4 \mathrm{~nm})$. Thus we hypothesize that the individual PLMA ${ }_{39}-\mathrm{PBzMA}_{97}$ chains interact with the larger $\mathrm{PLMA}_{39}-\mathrm{PBzMA}_{294}$ nanoparticles to form the hybrid nanoparticles. Time-resolved SAXS studies confirm that the evolution in copolymer morphology occurs on relatively short time scales (within $20 \mathrm{~min}$ at $150{ }^{\circ} \mathrm{C}$ ) and involves weakly anisotropic intermediate species. Moreover, weakly anisotropic nanoparticles can be obtained as a final copolymer morphology over a restricted range of compositions (e.g. for $\mathrm{PLMA}_{39}-\mathrm{PBzMA}_{97}$ volume fractions of 0.20-0.35) when heating dilute dispersions of such binary nanoparticle mixtures up to $150{ }^{\circ} \mathrm{C}$. A mechanism involving both chain expulsion/insertion and micelle fusion/fission is proposed to account for these unexpected observations.

\section{Introduction}

Block copolymer self-assembly has underpinned numerous remarkable advances in the field of materials science over the past two decades or so. ${ }^{1}$ For example, self-assembly in the solid state has led to the development of block copolymer nanolithography for information storage and nanofiltration. ${ }^{2-5}$ Similarly, self-assembly in solution has led to potential applications in microfluidic devices, ${ }^{6}$ automobile lubricants, ${ }^{7}$ viscosity modifiers, ${ }^{\mathbf{8}}$ stem cell storage media ${ }^{9}$ and drug delivery. ${ }^{\mathbf{1 0}}$

The first example of well-defined diblock copolymers were prepared by anionic polymerization. ${ }^{\mathbf{1 1}}$ This living polymerization technique allows the synthesis of copolymer chains with very narrow molecular weight distributions but it is extremely sensitive to protic impurities and is applicable to only a narrow range of vinyl monomers. Fortunately, developments in the field of controlled radical polymerization, particularly reversible
${ }^{a}$ Department of Chemistry, University of Sheffield, Dainton Building, Brook Hill, Sheffield, South Yorkshire S3 7HF, UK. E-mail: o.mykhaylyk@sheffield.ac.uk; s.p. armes@sheffield.ac.uk

${ }^{b}$ Lubrizol Ltd, Nether Lane, Hazelwood, Derbyshire, DE56 4AN, UK

$\dagger$ Electronic supplementary information (ESI) available: Materials and method section with protocols for nanoparticle syntheses and characterization; SAXS models and input parameters; SAXS patterns of $\mathrm{PLMA}_{39}-\mathrm{PBzMA}_{97}$ and $\mathrm{PLMA}_{39}-\mathrm{PBzMA}_{294}$ spheres at various temperatures with fitting parameters; fitted SAXS pattern for a PLMA $_{39}$ macro-CTA; SAXS fitting parameters for hybrid spheres; fitted SAXS patterns for hybrid nanoparticles obtained at various volume fractions with corresponding input parameters. See DOI: 10.1039/d0sc00569j 
addition-fragmentation chain transfer (RAFT) polymerization, enable the preparation of many functional block copolymers under much less demanding reaction conditions. ${ }^{12-19}$

Traditionally, block copolymer self-assembly in solution has been performed via post-polymerization processing using a solvent-switch method. ${ }^{1}$ A much more convenient approach for the preparation of block copolymer nanoparticles involves RAFT-mediated polymerization-induced self-assembly (PISA). ${ }^{17,20-28}$ In essence, PISA involves chain extension of a soluble precursor block with a second insoluble block. Block copolymer self-assembly occurs in situ once the growing latter block reaches a critical mean degree of polymerization (DP). Polymerization continues thereafter within monomer-swollen nanoparticles. The high local monomer concentration leads to a rate acceleration while the unreacted monomer acts as a processing aid (or co-solvent). Given a sufficiently short steric stabilizer block, either spheres, worms/cylinders or vesicles obtained depending on the relative volume fractions of each block. In contrast, longer steric stabilizer blocks invariably lead to kinetically-trapped spheres, because sphere-sphere fusion is impeded. ${ }^{20-22}$

It is well-known that thermal annealing can lead to the exchange of diblock copolymer chains between nanoparticles in polar ${ }^{29-35}$ and non-polar media, as shown by both Lodge and coworkers and Growney et al. ${ }^{36-43}$ Two mechanisms have been suggested for this phenomenon: (i) a chain expulsion/insertion mechanism and (ii) a micelle fusion/fission mechanism. ${ }^{41,44-48}$ Theoretical studies by Halperin ${ }^{45,46}$ and experimental observations made by Lund and co-workers ${ }^{31,35}$ and Lodge and coworkers ${ }^{36-39,41,49,50}$ suggest that the former mechanism is much more likely for diblock copolymer micelles (a.k.a. stericallystabilized nanoparticles). On the other hand, Armes and coworkers have suggested that particle-particle fusion is likely to play an important role during certain PISA syntheses, for which unreacted monomer plays an important processing role as a co-solvent. Indeed, such a fusion mechanism seems to be the most likely explanation for the in situ self-assembly of highly anisotropic diblock copolymer worms, whose formation is favored at higher copolymer concentrations..$^{21,22,51-53}$ In principle, chain expulsion/insertion and micelle fusion/fission could each play important roles during RAFT PISA.

Herein we examine copolymer exchange for various binary mixtures of dilute copolymer dispersions comprising poly(lauryl methacrylate)-poly(benzyl methacrylate) $\left(\mathrm{PLMA}_{39^{-}}\right.$ $\operatorname{PBzMA}_{x}$ ) spherical nanoparticles with mean core diameters of $21 \pm 2 \mathrm{~nm}$ and $48 \pm 5 \mathrm{~nm}$, respectively. These kineticallytrapped spheres were prepared in $n$-dodecane via RAFTmediated PISA using a previously reported protocol; ${ }^{22,53}$ the core-forming PBzMA $x$ block DP $(x)$ was either 97 or 294 . Variable temperature small-angle X-ray scattering (SAXS) studies provide valuable insights regarding the behavior of these nanoparticles when annealed separately at $150{ }^{\circ} \mathrm{C}$ with regard to their degree of core solvation and change in aggregation number. Annealing binary mixtures of this pair of nanoparticles over a wide range of relative volume fractions leads to the formation of a series of new hybrid nanoparticles. Time-resolved SAXS studies are used to examine the time scale for this hybridization process and also to examine its mechanism. This powerful characterization technique provides compelling evidence for the presence of weakly anisotropic intermediate species during the in situ evolution in copolymer morphology. Under certain conditions, weakly anisotropic nanoparticles can also be obtained as the final copolymer morphology from such hybridization experiments.

\section{Results and discussion}

\section{Preparation and characterization of PLMA-PBzMA spheres in n-dodecane}

Well-defined PLMA ${ }_{39}-\mathrm{PBzMA}_{x}$ spherical nanoparticles (where $x$ is either 97 or 294 ) were prepared at $20 \% \mathrm{w} / \mathrm{w}$ solids in $n$-dodecane using a well-established RAFT-mediated PISA protocol. ${ }^{22,53}$ A PLMA $_{39}$ precursor was chain-extended with BzMA monomer, with micellar nucleation occurring once a critical PBzMA core DP was attained (Scheme 1). High BzMA monomer conversions (>97\%) were achieved in both cases according to ${ }^{1} \mathrm{H}$ NMR spectroscopy analysis of the crude reaction mixtures, after dilution with sufficient $\mathrm{CDCl}_{3}$ to ensure nanoparticle dissolution (Fig. S1 and eqn (S1) in the ESI $\dagger$ ). Both types of diblock copolymer nanoparticles were dissolved in THF prior to GPC analysis (Fig. S2 $\dagger$ ). Relatively narrow unimodal molecular weight distributions were obtained $\left(M_{\mathrm{w}} / M_{\mathrm{n}} \leq 1.20\right)$, suggesting pseudo-living character for this RAFT dispersion polymerization. A clear shift to lower retention time for each diblock copolymer compared to that for the $\mathrm{PLMA}_{39}$ precursor indicated a high blocking efficiency in each case. For closelyrelated PISA formulations, Fielding et al. reported that targeting higher PBzMA DPs simply led to progressively larger kinetically-trapped spherical nanoparticles when utilizing a sufficiently long PLMA stabilizer block. ${ }^{22}$ In the present study, SAXS patterns recorded at $25{ }^{\circ} \mathrm{C}$ for a $1.0 \% \mathrm{w} / \mathrm{w}$ dispersion of $\mathrm{PLMA}_{39}-\mathrm{PBzMA}_{x}$ nanoparticles could be fitted using a spherical micelle model $^{54,55}$ (eqn (S2)-(S13) and Table S1 $\dagger$ ). This approach indicated spherical nanoparticle core diameters of $21 \pm 2 \mathrm{~nm}$ and $48 \pm 5 \mathrm{~nm}$ for the $\mathrm{PLMA}_{39}-\mathrm{PBzMA}_{97}$ and $\mathrm{PLMA}_{39}-\mathrm{PBzMA}_{294}$ spheres, respectively (Fig. S3, Tables S2 and S3†). The mean radius of gyration for the $\mathrm{PLMA}_{39}$ stabilizer chains was approximately $2.0 \mathrm{~nm}$ in both cases. This is consistent with the radius of gyration of $1.9 \mathrm{~nm}$ obtained by fitting the SAXS pattern recorded for a $1.0 \% \mathrm{w} / \mathrm{w}$ solution of PLMA39 homopolymer in $n$-dodecane (Fig. S4†) using the Debye function. ${ }^{56}$ SAXS pattern for the larger nanoparticles revealed an additional diffuse peak at $q \sim 0.8 \mathrm{~nm}^{-1}$ (Fig. S3B $\dagger$ ). We account for this feature by including a Gaussian function in the data fit. This high $q$ feature indicates a length scale of approximately $8 \mathrm{~nm}$, which corresponds to twice the radius of gyration of the PBzMA chains $(\sim 4$ $\mathrm{nm}$ ) within the nanoparticle cores.

\section{Variable temperature SAXS analysis of spherical nanoparticles}

It is well-known that copolymer chain exchange can occur between spherical diblock copolymer nanoparticles in nonpolar media at elevated temperature. Moreover, such exchange is sensitive to both temperature and the DP of the 


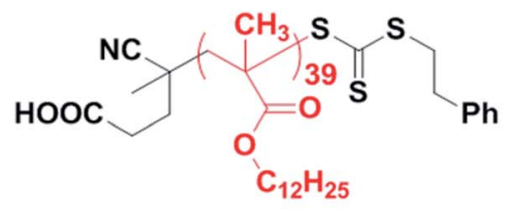

$\mathrm{PLMA}_{39}$ macro-CTA

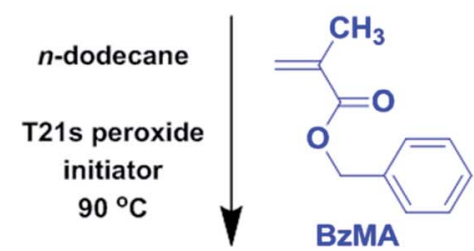<smiles>[X]C(C)(CC(C)(C)C(C)(C)C(=O)OCc1ccccc1)C(C)(C)C(=O)OCc1ccccc1</smiles>

$\mathrm{PLMA}_{39}-\mathrm{PBzMA}_{\mathrm{x}}$ (where $\mathrm{x}=97$ or 294 )



Scheme 1 A PLMA 39 precursor block is chain-extended with BzMA monomer in $n$-dodecane via RAFT-mediated PISA to form stericallystabilized spherical nanoparticles. SAXS analysis (see Fig. S3, Tables S2 and S3 in the ESI + ) indicated that final PBzMA DPs of 97 and 294 produced mean core diameters of $21 \pm 2 \mathrm{~nm}$ and $48 \pm 5 \mathrm{~nm}$, respectively.

core-forming block. ${ }^{38,41}$ It is also known that the cores of PLMAPBzMA nano-objects become progressively more solvated with hot solvent at elevated temperature. ${ }^{8,53}$ It seems likely that the greater chain mobility associated with this solvent plasticization facilitates the chain expulsion/insertion mechanism, making the thermally-activated redistribution of copolymer chains more likely. Herein, variable temperature SAXS was used to study the degree of core solvation and integrity of $\mathrm{PLMA}_{39}-\mathrm{PBzMA}_{97}$ and $\mathrm{PLMA}_{39}-\mathrm{PBzMA}_{294}$ spheres at elevated temperature. In an initial series of experiments, dispersions of both types of nanoparticles were diluted in turn to $1.0 \% \mathrm{w} / \mathrm{w}$ using $n$-dodecane and scattering patterns were recorded in each case during a $25-150-25{ }^{\circ} \mathrm{C}$ thermal cycle (Fig. S3†). Heating $\mathrm{PLMA}_{39}-\mathrm{PBzMA}_{97}$ spheres alone led to a progressive change in the scattering pattern, which returned to its original form after cooling from $150{ }^{\circ} \mathrm{C}$ to $25^{\circ} \mathrm{C}$ (Fig. S3A $\dagger$ ). In contrast, heating the larger $\mathrm{PLMA}_{39}-\mathrm{PBzMA}_{294}$ spheres up to $150{ }^{\circ} \mathrm{C}$ produced almost no discernible change in the scattering pattern (Fig. S3B $\dagger$ ). SAXS patterns recorded for the $\mathrm{PLMA}_{39^{-}}$ $\mathrm{PBzMA}_{97}$ and $\mathrm{PLMA}_{39}-\mathrm{PBzMA}_{294}$ spheres at various temperatures were fitted to a well-known spherical micelle model with an additional Debye function to account for a minor fraction of molecularly-dissolved $\mathrm{PLMA}_{39}-\mathrm{PBzMA}_{x}$ chains (see eqn (S2)(S13) in the ESI $\dagger$ ). ${ }^{54-56}$ Satisfactory data fits could be obtained for both types of nanoparticles by assuming that the change in mass density (which affects both the scattering length density and the individual block volumes) for the PLMA corona block and the PBzMA core-forming block was equal to that reported by Fetters and co-workers for poly(n-butyl methacrylate) and polystyrene, respectively (Table S1 $\dagger$ ). ${ }^{57}$ SAXS analysis indicated minimal change in mean diameter for both types of spherical nanoparticles on heating up to $150{ }^{\circ} \mathrm{C}$ (Fig. 1, Tables S2 and S3 in the ESI $\dagger$ ). However, a progressive increase in the solvent volume fraction $\left(X_{\text {sol }}\right)$ within the nanoparticle cores was


Fig. 1 Mean nanoparticle core diameter and solvent volume fraction $\left(X_{\text {sol }}\right)$ determined for (A) PLMA $39-P_{38}$ PA $_{97}$ and (B) PLMA $39-P_{32}$ PA $_{294}$ spheres at various temperatures using SAXS analysis (Tables S2 and $\mathrm{S} 3, \uparrow$ respectively). The core diameter remains approximately constant for both types of nanoparticles when heated up to $150{ }^{\circ} \mathrm{C}$. However, progressively higher $X_{\text {sol }}$ values are observed on heating $\mathrm{PLMA}_{39}-$ $\mathrm{PBzMA}_{97}$ spheres. The original $X_{\text {sol }}$ value of approximately zero is obtained for this system on returning to $25^{\circ} \mathrm{C}$. In contrast, $\mathrm{PLMA}_{39}-$ $\mathrm{PBzMA}_{294}$ spheres exhibit no discernible change in $X_{\text {sol }}$ on heating up to $150{ }^{\circ} \mathrm{C}$. [N.B. Error bars correspond to the standard deviation in the mean nanoparticle diameter.] 
observed when heating the smaller $\mathrm{PLMA}_{39}-\mathrm{PBzMA}_{97}$ spheres. Moreover, a drastic reduction in nanoparticle concentration from 0.67 to $0.45 \% \mathrm{v} / \mathrm{v}$ was observed on heating to $150{ }^{\circ} \mathrm{C}$. To account for this observation, the Debye function ${ }^{56}$ was used to include a population of molecularly-dissolved $\mathrm{PLMA}_{39^{-}}$ PBzMA $_{97}$ chains in the data fit, such that this population becomes progressively larger at higher temperatures. Returning to $25{ }^{\circ} \mathrm{C}$ led to complete desolvation of the nanoparticle cores and the final data fit at this temperature indicated that only a minor population of $\mathrm{PLMA}_{39}-\mathrm{PBzMA}_{97}$ chains remained molecularly dissolved (Table S2†).

In contrast to the smaller nanoparticles, data fits obtained for the larger $\mathrm{PLMA}_{39}-\mathrm{PBzMA}_{294}$ spheres indicated minimal change in their mean diameter and degree of core solvation when thermally annealed at $150{ }^{\circ} \mathrm{C}$. Moreover, the SAXS data fits indicate a rather more subtle increase in the relative concentration of the molecularly-dissolved PLMA $_{39}-\mathrm{PBzMA}_{294}$ chains compared to that of the spherical nanoparticles (Table S3†). In summary, the above variable temperature SAXS experiments indicate that the cores of the larger spheres are much less plasticized by hot solvent compared to the smaller spheres, which means that the former nanoparticles are much less likely to undergo dissociation (i.e. expulsion of individual copolymer chains).

The mean core diameter for the highly solvated $\mathrm{PLMA}_{39^{-}}$ $\mathrm{PBzMA}_{97}$ nanoparticles at $150{ }^{\circ} \mathrm{C}$ is comparable to that of the same non-solvated nanoparticles at $25{ }^{\circ} \mathrm{C}$. This implies a significant reduction in the volume-average aggregation number, $N_{\text {agg }}$, (i.e. the mean number of copolymer chains per nanoparticle) at elevated temperature. This parameter was calculated using eqn (1):

$$
N_{\text {agg }}=\left(1-X_{\text {sol }}\right) \frac{\frac{4}{3} \pi R^{3}}{V_{\mathrm{s}}}
$$

where $R$ is the nanoparticle core radius and $V_{\mathrm{s}}$ is the volume occupied by a single PBzMA core-forming block. The calculated $N_{\text {agg }}$ values are plotted against temperature for both PLMA $_{39^{-}}$ PBzMA $_{97}$ and PLMA $_{39}-$ PBzMA $_{294}$ spheres (Fig. 2). The mean $N_{\text {agg }}$ for the former spheres was significantly reduced from 205 at $25{ }^{\circ} \mathrm{C}$ to 142 at $150{ }^{\circ} \mathrm{C}$, which is consistent with the formation of a secondary population of molecularly-dissolved diblock copolymer chains. Returning to $25{ }^{\circ} \mathrm{C}$ led to the formation of spheres with a slightly lower $N_{\text {agg }}$ (171) than the original nanoparticles. It seems likely that annealing $\mathrm{PLMA}_{39}-\mathrm{PBzMA}_{97}$ spheres at $150{ }^{\circ} \mathrm{C}$ enables the diblock copolymer chains to rearrange to form spheres that lie closer to the thermodynamically-preferred size compared to the original spheres formed via RAFT-mediated PISA at $90^{\circ} \mathrm{C}$. Furthermore, these results suggest that a small fraction of molecularlydissolved $\mathrm{PLMA}_{39}-\mathrm{PBzMA}_{97}$ chains remain dissolved after returning to $25{ }^{\circ} \mathrm{C}$. In contrast, the minimal change in nanoparticle core diameter (along with the lack of solvation) observed for the larger $\mathrm{PLMA}_{39}-\mathrm{PBzMA}_{294}$ spheres during thermal annealing suggests an approximately constant $N_{\text {agg }}$ during a $25-150-25{ }^{\circ} \mathrm{C}$ thermal cycle. In summary, SAXS analysis suggests that the smaller $\mathrm{PLMA}_{39}-\mathrm{PBzMA}_{97}$ spheres



Fig. 2 Temperature dependence for the mean aggregation number $\left(N_{\text {agg }}\right)$ determined by SAXS analysis of $1.0 \% \mathrm{w} / \mathrm{w}$ dispersions of (black) $\mathrm{PLMA}_{39}-\mathrm{PBzMA}_{97}$ and (red) $\mathrm{PLMA}_{39}-\mathrm{PBzMA}_{294}$ spheres at various temperatures. The former spheres exhibit a significant reduction in $N_{\text {agg }}$ on heating, while the latter exhibit minimal change in aggregation number.

undergo dissociation to form molecularly-dissolved copolymer chains at $150{ }^{\circ} \mathrm{C}$ whereas the larger $\mathrm{PLMA}_{39}-\mathrm{PBzMA}_{294}$ spheres remain relatively stable under such conditions. It is perhaps noteworthy that this interpretation is consistent with the strong DP and diameter dependence for copolymer chain exchange reported by Lodge and co-workers. ${ }^{38,41,58}$

\section{Hybridization of PLMA $_{39}-$ PBzMA $_{97}$ and PLMA $_{39}-$ PBzMA $_{294}$ spheres}

An equivolume binary mixture of $\mathrm{PLMA}_{39}-\mathrm{PBzMA}_{97}$ and $\mathrm{PLMA}_{39}-\mathrm{PBzMA}_{294}$ spheres at $1.0 \% \mathrm{w} / \mathrm{w}$ solids was heated up to $150{ }^{\circ} \mathrm{C}$ for $1 \mathrm{~h}$ to examine the possibility of thermally-activated exchange of copolymer chains between such nanoparticles. In initial experiments, thermal annealing of an equivolume binary mixture of these two types of nanoparticles leads to the formation of well-defined spherical nanoparticles of intermediate mean diameter (see Scheme 2).

TEM analysis confirmed a well-defined spherical morphology for both types of nanoparticles prior to heating. As expected, the binary dispersion exhibited two distinct populations prior to thermal annealing. Interestingly, TEM analysis of this binary mixture after heating to $150{ }^{\circ} \mathrm{C}$ for $1 \mathrm{~h}$ indicated a single population of spherical nanoparticles exhibiting an intermediate mean particle diameter (Fig. 3). These TEM observations were supported by SAXS experiments performed at



Scheme 2 An equivolume binary mixture of $\mathrm{PLMA}_{39}-\mathrm{PBzMA}_{97}$ and $\mathrm{PLMA}_{39}-\mathrm{PBzMA}_{294}$ spheres at $1.0 \% \mathrm{w} / \mathrm{w}$ solids forms spheres of intermediate size when annealed at $150{ }^{\circ} \mathrm{C}$ for $1 \mathrm{~h}$. 



Fig. 3 SAXS patterns (and corresponding TEM images) recorded at $25{ }^{\circ} \mathrm{C}$ for $1.0 \% \mathrm{w} / \mathrm{w}$ dispersions of: (A) PLMA ${ }_{39}-\mathrm{PBzMA}_{294}$ spheres and (B) $\mathrm{PLMA}_{39}-\mathrm{PBzMA}_{97}$ spheres; (C) a $1.0 \% \mathrm{w} / \mathrm{w}$ equivolume binary mixture of these two initial dispersions prior to thermal annealing and (D) the final hybrid nanoparticles formed after thermal annealing of the same binary mixture at $150^{\circ} \mathrm{C}$ for $1 \mathrm{~h}$. White traces indicate the best fits to the data obtained when using a spherical micelle model. ${ }^{54-56}$ Scale bars shown in TEM images correspond to $100 \mathrm{~nm}$.

ambient temperature (Scheme 2 and Fig. 3). According to general scattering theorems, the scattering intensity in the low $q$ region of the X-ray scattering pattern $\left(q \sim 0 \AA^{-1}\right)$ is proportional to the nanoparticle volume. ${ }^{59}$ Thus larger nanoparticles cause stronger X-ray scattering in this regime. Furthermore, the gradient of the pattern in this low $q$ region is sensitive to the nanoparticle shape. For example, a zero gradient indicates the presence of isotropic spheres. In contrast, anisotropic nanoparticles exhibit a negative gradient in this low $q$ region. Thus, platelets/disks or vesicles possess a gradient of -2 , while long thin rods are characterized by a gradient of -1 (with less anisotropic rods typically possessing gradients ranging between zero and -1$).^{60}$

The scattering patterns recorded for the two initial dispersions, their equivolume binary mixture and the final hybrid nanoparticles exhibited approximate zero gradients at low $q$, which indicates the presence of spherical non-interacting nanoparticles in each case. Moreover, the scattering pattern recorded for the initial binary mixture of nanoparticles could be satisfactorily fitted using the known size distribution for each component by simply varying the relative concentrations of the two types of nanoparticles (Table S4 $\dagger$ ). The SAXS pattern recorded for the annealed binary dispersion is consistent with the TEM images shown in Fig. 3: the local minimum in the scattering curve clearly falls between the two minima observed for the original large and small spherical nanoparticles, which confirms that hybrid spherical nanoparticles with an intermediate mean diameter are obtained after heat treatment. The scattering pattern recorded for these hybrid nanoparticles was fitted to the same spherical micelle model using a mean PBzMA core volume calculated from the known proportions of the two nanoparticle populations (Table S4†). Assuming complete entropic mixing, this fit to the scattering curve gave a mean volume-average core diameter of $36 \pm 3 \mathrm{~nm}$, which is consistent with the number-average core diameter of $34 \pm 3 \mathrm{~nm}$ estimated from TEM analysis (Fig. 3D). This confirms that the dimensions of the hybrid nanoparticles lie between those of the two initial nanoparticle dispersions.

\section{In situ SAXS studies of the kinetics of nanoparticle hybridization}

To explore the nanoparticle hybridization mechanism, in situ SAXS measurements were performed while heating a $1.0 \% \mathrm{w} / \mathrm{w}$ dispersion comprising an equivolume binary mixture of $\mathrm{PLMA}_{39}-\mathrm{PBzMA}_{97}$ and $\mathrm{PLMA}_{39}-\mathrm{PBzMA}_{294}$ spheres up to $150{ }^{\circ} \mathrm{C}$ (Fig. 4). A scattering pattern for the initial binary mixture was recorded at $20{ }^{\circ} \mathrm{C}$, with two distinct minima representing the bimodal nature of the initial nanoparticle dispersion (Fig. 3). After heating up to $150{ }^{\circ} \mathrm{C}$ at $30{ }^{\circ} \mathrm{C} \mathrm{min}{ }^{-1}$, the first scattering



Fig. 4 In situ SAXS patterns recorded when heating a $1.0 \% \mathrm{w} / \mathrm{w}$ nanoparticle dispersion comprising a $1: 1 \mathrm{v} / \mathrm{v}$ binary mixture of $21 \pm 2$ $\mathrm{nm} \mathrm{PLMA} A_{39}-\mathrm{PBzMA}_{97}$ and $48 \pm 5 \mathrm{~nm} \mathrm{PLMA} 39-\mathrm{PBzMA}_{294}$ spheres up to $150{ }^{\circ} \mathrm{C}$ at a heating rate of $30^{\circ} \mathrm{C} \mathrm{min}^{-1}$. As expected, two minima are discernible in the initial pattern recorded for this binary mixture at $20{ }^{\circ} \mathrm{C}$. However, the final scattering pattern obtained after annealing for $20 \mathrm{~min}$ at $150{ }^{\circ} \mathrm{C}$ shows two minima (denoted $q_{1}$ and $q_{2}$ ) that correspond to a single population of spherical nanoparticles of intermediate diameter (36 $\pm 4 \mathrm{~nm}$ ). 
pattern recorded at this temperature also exhibited these two minima. However, maintaining this dilute binary dispersion at $150{ }^{\circ} \mathrm{C}$ produced a low $q$ gradient of -0.55 within $7.0 \mathrm{~min}$. Thereafter, a low $q$ gradient of approximately zero was again observed (within a further $12.5 \mathrm{~min}$ ). The final scattering pattern acquired after thermal annealing for $20 \mathrm{~min}$ at $150{ }^{\circ} \mathrm{C}$ exhibited two minima that correspond to a single population of spherical nanoparticles (Scheme 2). [N.B. For these two features, we find that $q_{1} R=4.49$ and $q_{2} R=7.73$, where $R$ corresponds to the core radius of the final hybrid spherical nanoparticles, Fig. 4.] Thus $R$ is calculated to be approximately $18 \mathrm{~nm}$ in each case, which indicates a mean particle diameter of $\sim 36 \mathrm{~nm}$. In summary, these in situ SAXS studies strongly suggest that the transformation of the initial binary mixture of spheres (Fig. 3C) into a single population of spheres of intermediate diameter (Fig. 3D) involves weakly anisotropic transient species.

The scattered X-ray intensity at an arbitrary $q$ value of 0.019 $\mathrm{nm}^{-1}$ (Fig. 5A), and the low $q$ gradient in the $0.019 \mathrm{~nm}^{-1}<q<$ $0.035 \mathrm{~nm}^{-1}$ interval (Fig. 5B) were plotted against time to further investigate the mechanism of formation of the final hybrid nanoparticles. Using a heating rate of $30{ }^{\circ} \mathrm{C} \mathrm{min}^{-1}$, the final temperature of $150{ }^{\circ} \mathrm{C}$ was achieved within 4.3 min during these in situ SAXS experiments. Inspecting Fig. 5, both the scattered intensity and the low $q$ gradient begin to change just below $150{ }^{\circ} \mathrm{C}$, suggesting that nanoparticle hybridization has already commenced prior to the target temperature being attained. A pronounced maximum in scattered X-ray intensity is observed at around 7.0 min (Fig. 5A), which roughly corresponds to the formation of the most anisotropic transient species as judged by the change in the low $q$ observed gradient (Fig. 5B). It should also be noted that the initial scattered X-ray intensity of $199 \mathrm{~cm}^{-1}$ is higher than the final intensity $\left(159 \mathrm{~cm}^{-1}\right)$ (Fig. $\left.5 \mathrm{~A}\right)$. This is understandable because the X-ray scattering at low $q$ is proportional to the volume of the scattering objects. As a result, X-ray scattering from the original equivolume binary mixture of large and small nanoparticles should be dominated by the former population, which also scatter more strongly than the final spheres of intermediate size [this latter point is readily illustrated by simple calculation, i.e. $\left.\left(48^{3}+21^{3}\right) / 2>36^{3}\right]$.

The progressive change in the low $q$ gradient during thermal annealing indicates the formation of weakly anisotropic species (mean aspect ratio $=2-3$ ). This unexpected observation suggests that the change in copolymer morphology is unlikely to simply involve expulsion of $\mathrm{PLMA}_{39^{-}}$ $\mathrm{PBzMA}_{97}$ copolymer chains from the smaller nanoparticles and their subsequent insertion into the larger $\mathrm{PLMA}_{39}-\mathrm{PBzMA}_{294}$ nanoparticles. This is because such a copolymer chain exchange mechanism should simply reduce the diameter of the smaller nanoparticles while increasing that of the larger nanoparticles. ${ }^{61}$ Given that a spherical morphology has the lowest possible surface area per unit mass, it is difficult to envisage how such mass transport could result in the formation of non-isotropic nanoparticles. It seems much more likely that the transient anisotropic species are instead obtained via a fusion/fission process, despite the relatively low copolymer concentration $(1.0 \% \mathrm{w} / \mathrm{w})$ used for these thermal annealing
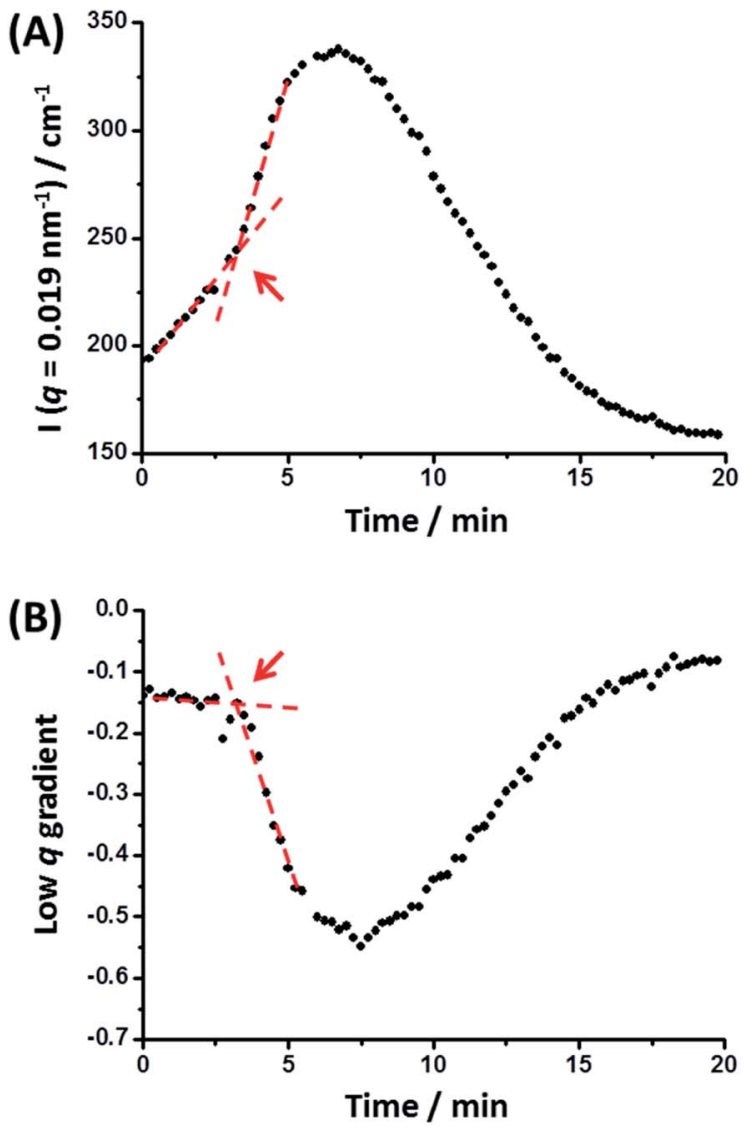

Fig. 5 (A) Variation in scattered X-ray intensity, $I(q)$, at an arbitrary $q$ value of $0.019 \mathrm{~nm}^{-1}$ and (B) change in the low $q$ gradient (for $0.019 \mathrm{~nm}^{-1}$ $<q<0.035 \mathrm{~nm}^{-1}$ ) over time recorded during an in situ SAXS experiment performed at $150{ }^{\circ} \mathrm{C}$ whereby spherical nanoparticles of intermediate diameter $(36 \pm 4 \mathrm{~nm})$ are generated from a dilute $1: 1 \mathrm{v} / \mathrm{v}$ binary mixture of spherical nanoparticles with initial volume-average diameters of $21 \pm$ $2 \mathrm{~nm}$ and $48 \pm 5 \mathrm{~nm}$, respectively (see Fig. 4 caption for further experimental details). The red lines and arrows indicate that a significant change in both the low $q$ gradient and the scattered X-ray intensity occurs after $3.2 \mathrm{~min}$, which corresponds to approximately $116^{\circ} \mathrm{C}$.

experiments. However, given that the extent of core solvation and mean aggregation number strongly depend on the DP of the core-forming block (Fig. 1 and 2), relatively few of the longer $\mathrm{PLMA}_{39}-\mathrm{PBzMA}_{294}$ chains are expected to be expelled from the larger nanoparticles at $150{ }^{\circ} \mathrm{C}$. Actually, the gradual increase in X-ray scattering intensity at $q=0.019 \mathrm{~nm}^{-1}$ and the low $q$ gradient of approximately zero indicates that the initial nanoparticles become progressively larger at $150{ }^{\circ} \mathrm{C}$ while maintaining their spherical morphology (Fig. 5, red lines and arrows). Thus, this observation suggests that the expelled $\mathrm{PLMA}_{39}-\mathrm{PBzMA}_{97}$ chains are simply incorporated within the larger $\mathrm{PLMA}_{39}-\mathrm{PBzMA}_{294}$ spheres for the first $3.2 \mathrm{~min}$.

However, a significant increase in X-ray scattering intensity is observed after $3.2 \mathrm{~min}$, which is accompanied by an abrupt change in the low $q$ gradient. These observations are consistent with the formation of larger, weakly anisotropic nanoparticles, e.g. dimers/trimers. The subsequent reduction in X-ray scattering intensity and return to a low $q$ gradient of 
approximately zero indicate the gradual evolution of these transient species into the final isotropic spheres of intermediate core diameter. Presumably, this latter process is driven by the minimization of surface free energy (see below for a proposed mechanism).

\section{Effect of varying the relative volume fraction of small nanoparticles on copolymer morphology}

Varying proportions of small $\mathrm{PLMA}_{39}-\mathrm{PBzMA}_{97}$ spheres were annealed with large $\mathrm{PLMA}_{39}-\mathrm{PBzMA}_{294}$ spheres in an attempt to produce the anisotropic intermediate species as a final hybrid copolymer morphology. These binary mixtures were prepared at various volumetric ratios at $20 \% \mathrm{w} / \mathrm{w}$ solids, then diluted to $1.0 \% \mathrm{w} / \mathrm{w}$ using $n$-dodecane and heated to $150{ }^{\circ} \mathrm{C}$ for $1 \mathrm{~h}$. $\mathrm{PLMA}_{39}-\mathrm{PBzMA}_{97}$ volume fractions ranging from 0.05 to 0.50 were examined in these nanoparticle fusion experiments. Indeed, TEM analysis of the annealed dispersions revealed formation of the anticipated weakly anisotropic nanoparticles (mean aspect ratio $=2-3$ ) when using $\mathrm{PLMA}_{39}-\mathrm{PBzMA}_{97}$ volume fractions of between 0.20 and 0.35 (Fig. 6). However, it is perhaps worth emphasizing that such species always co-exist with a variable population of spheres.

Further insight regarding the formation of these kinetically stable hybrid nanoparticles was obtained by SAXS analysis (Fig. S5†). Scattering patterns for these dispersions were recorded at $1.0 \% \mathrm{w} / \mathrm{w}$ solids after thermal annealing at this concentration. The structure factor can be assumed to be unity for such dispersions and the approximate zero gradient observed at low $q$ indicated a spherical morphology in most cases. However, a non-zero gradient was observed at low $q$ when using $\mathrm{PLMA}_{39}-\mathrm{PBzMA}_{97}$ volume fractions of $0.20,0.25$, 0.30 or 0.35 , indicating the presence of anisotropic nanoparticles. Moreover, these scattering patterns could not be fitted using a spherical micelle model (eqn (S2)-(S13) in the ESI) (Fig. S5†). These observations are consistent with TEM studies of these four thermally-annealed dispersions, for which a variable population of weakly anisotropic nanoparticles was observed at $20{ }^{\circ} \mathrm{C}$ (Fig. 6). These SAXS patterns were further analyzed by calculating nanoparticle dimensions (i.e. either the mean sphere diameter or a cross-sectional diameter for the dimer/trimer species) from the $q$ value of the first minimum (particle dimension $=4.49 / q_{\text {min }}$, where 4.49 corresponds to the first minimum for the spherical form factor), and plotting such sizes against the corresponding $\mathrm{PLMA}_{39}-\mathrm{PBzMA}_{97}$ volume fraction (Fig. 7). A modest monotonic increase in sphere diameter was observed for $\mathrm{PLMA}_{39^{-}}$ $\mathrm{PBzMA}_{97}$ volume fractions up to 0.15 . At higher volume fractions (0.20-0.35), a population of weakly anisotropic transient particles is indicated by the gradual reduction in mean particle dimension, i.e. the effective cross-section of the dimers/ trimers (Fig. 7). Finally, using a higher proportion of small $\mathrm{PLMA}_{39}-\mathrm{PBzMA}_{97}$ spheres (volume fraction $=0.40-0.50$ ) leads to a gradual reduction in the mean sphere diameter. In this case, it appears that there is a sufficiently high concentration of $\mathrm{PLMA}_{39}-\mathrm{PBzMA}_{97}$ chains to generate a single population of spheres of intermediate size, as observed in Fig. 3 and 6.



Fig. 6 TEM images recorded for the series of hybrid nanoparticles formed after annealing $1.0 \% \mathrm{w} / \mathrm{w}$ binary mixtures of $\mathrm{PLMA}_{39}-\mathrm{PBzMA}_{97}$ and $\mathrm{PLMA}_{39}-\mathrm{PBzMA}_{294}$ spheres at $150{ }^{\circ} \mathrm{C}$ for $1 \mathrm{~h}$. Caption labels denote the volume fraction of the smaller PLMA ${ }_{39}-\mathrm{PBzMA}_{97}$ spheres used in these experiments. The first two images correspond to the initial spherical nanoparticles annealed individually at $1.0 \% \mathrm{w} / \mathrm{w}$ solids in control experiments. All scale bars correspond to $100 \mathrm{~nm}$. [N.B. The core diameters estimated from these TEM images are consistent with the corresponding SAXS studies, see Fig. 7].

\section{Proposed mechanism for nanoparticle hybridization}

Taking into consideration the TEM images and SAXS data discussed above, a tentative two-stage mechanism is proposed in 




Fig. 7 Change in nanoparticle dimensions (and morphology) observed when varying the volume fraction of $\mathrm{PLMA}_{39}-\mathrm{PBzMA}_{97}$ nanoparticles in thermal annealing experiments conducted at $150{ }^{\circ} \mathrm{C}$ using a $1.0 \% \mathrm{w} / \mathrm{w}$ binary mixture of $\mathrm{PLMA}_{39}-\mathrm{PBzMA}_{97}$ and $\mathrm{PLMA}_{39}-$ $\mathrm{PBzMA}_{294}$ nanoparticles in $n$-dodecane (see TEM images in Fig. 6 and corresponding SAXS patterns in Fig. S5†). Hybrid nanoparticle dimensions (i.e. either sphere diameter or mean cross-sectional diameter of the dimers/trimers) were calculated from the $q$ value for the first minima in the corresponding SAXS patterns (particle dimension $=4.49 / q_{\mathrm{min}}$, where the numerical factor corresponds to the first minimum for a spherical form factor).

Scheme 3. In Stage 1, individual copolymer chains are expelled from the $\mathrm{PLMA}_{39}-\mathrm{PBzMA}_{97}$ nanoparticles at $150{ }^{\circ} \mathrm{C}$ and are reinserted into the larger $\mathrm{PLMA}_{39}-\mathrm{PBzMA}_{294}$ nanoparticles, which barely undergo any copolymer exchange under such conditions (see Fig. 1 and 2). This causes the latter nanoparticles to grow in size, which simply leads to the formation of larger hybrid spheres provided that the volume fraction of the smaller PLMA $_{39}-\mathrm{PBzMA}_{97}$ nanoparticles is less than 0.20. However, above this critical volume fraction a second series of events occurs (see Stage 2). Now the growing hybrid nanoparticles interact with one (or more) of the smaller, solventswollen spheres and undergo fusion and internal rearrangement to form weakly anisotropic intermediate species. The surfactant-like individual copolymer chains then adsorb onto and interact with these relatively unstable intermediates, which undergo fission to form at least two hybrid spheres of intermediate size. This hypothesis is consistent with the observations made during the in situ SAXS experiment (Fig. 4 and 5). In particular, the initial increase in scattered X-ray intensity at $q=0.019 \mathrm{~nm}^{-1}$ for the first 3.2 min corresponds to Stage 1, since the low $q$ gradient remains close to zero (indicating that only spheres are present). However, the scattered X-ray intensity then increases significantly with a concomitant reduction in the low $q$ gradient to -0.55 being observed at $7.0 \mathrm{~min}$. This indicates the formation of relatively large, weakly anisotropic intermediates. Such species can be obtained as a final morphology if the volume fraction of smaller $\mathrm{PLMA}_{39}-\mathrm{PBzMA}_{97}$ nanoparticles lies between 0.20 and 0.35 . However, at higher volume fractions, these smaller nanoparticles provide a sufficient quantity of surface-active individual copolymer chains to



Hybrid nanoparticles No further change if volume fraction of $21 \mathrm{~nm}$ diameter nanoparticles is less than $\mathbf{0 . 2 0}$



Scheme 3 Schematic representation of the two-stage mechanism proposed for the changes in copolymer morphology that are observed during thermal annealing of a binary mixture of $21 \pm 2 \mathrm{~nm}$ and $48 \pm$ $5 \mathrm{~nm}$ diblock copolymer spheres at $150^{\circ} \mathrm{C}$. Here, the $n, m$ and $p$ values refer to the number density of each type of nanoparticle. In Stage 1, the smaller $\mathrm{PLMA}_{39}-\mathrm{PBz} \mathrm{MA}_{97}$ spheres undergo partial dissociation to form copolymer chains, which then become incorporated into the larger spheres to produce hybrid spheres with a mean diameter greater than $48 \mathrm{~nm}$. If the volume fraction of these smaller spheres is less than 0.20 , this is the final copolymer morphology. However, using higher volume fractions of this component leads to Stage 2, whereby the $21 \mathrm{~nm}$ spheres undergo fusion with the larger hybrid spheres to form weakly anisotropic transient species. The latter then undergo fission - most likely mediated by incorporation of further PLMA $\mathrm{M}_{39}-\mathrm{PBzMA}_{97}$ chains to form spheres of intermediate size (e.g. $36 \mathrm{~nm}$ diameter). This mechanism is consistent with the SAXS data shown in Fig. 3-5 and 7 and the TEM images shown in Fig. 3 and 6.

interact with these anisotropic intermediates. This causes the latter species to undergo fission, which produces the final hybrid spheres of intermediate diameter. This hypothesis is consistent with a prior study by Chambon and co-workers, ${ }^{62}$ who found that diblock copolymer vesicles prepared via aqueous PISA could be disrupted to form much smaller spheres when exposed to an ionic surfactant. This fission event is responsible for the gradual reduction in the scattered X-ray intensity and the low $q$ gradient shown in Fig. 5 .

Revisiting the representative TEM images shown in Fig. 3, we calculate (see ESI $\dagger$ for further details) that approximately eleven small $(21 \pm 2 \mathrm{~nm})$ PLMA $_{39}-$ PBzMA $_{97}$ spherical nanoparticles interact with each large $(48 \pm 5 \mathrm{~nm}) \mathrm{PLMA}_{39}-\mathrm{PBzMA}_{294}$ sphere to form approximately four hybrid nanoparticles of $36 \mathrm{~nm}$ diameter. This calculation should be borne in mind when considering Scheme 3 (i.e. $n=11, m=1$ and $p \sim 4$ for the thermal annealing experiment described in Fig. 3). However, this rather rudimentary analysis suffers from poor statistics. Thus, we also reexamined the corresponding SAXS data obtained for the thermally-annealed nanoparticles. This enabled us to calculate a fusion ratio that was remarkably close to that obtained from the above TEM image analysis $(n \sim 9, m=1$ and $p$ $\sim 4$ ), see Table S6 in the ESI. $\dagger$ 
The driving force for the fusion process is likely to be the (partial) solvation of the nanoparticle cores by the ingress of hot solvent at elevated temperature. This inevitably leads to enhanced copolymer chain mobility, which in turn enhances the probability of micelle fusion. Indeed, we have recently published NMR evidence for such core solvation for a related PISA formulation in non-polar media. ${ }^{17}$ We suggest that using a higher annealing temperature $\left(>150{ }^{\circ} \mathrm{C}\right)$ is likely to affect the nanoparticle hybridization mechanism. Under such conditions, the $\mathrm{PLMA}_{39}-\mathrm{PBzMA}_{294}$ nanoparticles may also undergo dissociation to form molecularly-dissolved copolymer chains. Clearly, this hypothesis warrants further studies. Finally, one reviewer of this manuscript has suggested that the micelle fusion/fission mechanism demonstrated herein is unlikely to apply to nanoparticles (micelles) of equal size. This is because there is no difference in free energy in this case. This may explain why a copolymer chain exchange mechanism appears to operate in such instances. ${ }^{41}$

\section{Conclusions}

RAFT-mediated PISA was used to prepare sterically-stabilized $\mathrm{PLMA}_{39}-\mathrm{PBzMA}_{97}$ and $\mathrm{PLMA}_{39}-\mathrm{PBzMA}_{294}$ diblock copolymer spheres in $n$-dodecane, with mean core diameters of $21 \pm 2 \mathrm{~nm}$ and $48 \pm 5 \mathrm{~nm}$, respectively. Variable-temperature SAXS analysis of these dilute dispersions at $150{ }^{\circ} \mathrm{C}$ indicates substantial core solvation and a significant reduction in aggregation number for the smaller $\mathrm{PLMA}_{39}-\mathrm{PBzMA}_{97}$ spheres. In contrast, changes were much less pronounced for the larger $\mathrm{PLMA}_{39^{-}}$ PBzMA $_{294}$ spheres: after returning to $25{ }^{\circ} \mathrm{C}$, approximately the same core diameters were observed before and after thermal annealing.

Annealing an equivolume binary mixture of these two dispersions at $1.0 \% \mathrm{w} / \mathrm{w}$ led to the formation of hybrid nanoparticles with an intermediate mean core diameter of $36 \pm 3 \mathrm{~nm}$. This suggests that the copolymer chains expelled from the PLMA $_{39}-\mathrm{PBzMA}_{97}$ spheres exhibit surfactant-like behavior and cause fission of the larger $\mathrm{PLMA}_{39}-\mathrm{PBzMA}_{294}$ spheres. Moreover, in situ SAXS studies during such experiments revealed an upturn in the low $q$ gradient. This indicates the presence of weakly anisotropic transient species, which then induce fusion to form the final hybrid spherical nanoparticles of intermediate size.

Further insights regarding the nanoparticle hybridization mechanism were obtained by annealing a series of binary mixtures with varying proportions of $\mathrm{PLMA}_{39}-\mathrm{PBzMA}_{97}$ and $\mathrm{PLMA}_{39}-\mathrm{PBzMA}_{294}$ spheres at $1.0 \% \mathrm{w} / \mathrm{w}$ solids. Spherical nanoparticles of intermediate size were produced for $\mathrm{PLMA}_{39^{-}}$ PBzMA $_{97}$ volume fractions of between 0.40 and 0.50 . Interestingly, using volume fractions between 0.05 and 0.20 yielded spherical nanoparticles with slightly larger mean particle diameters than the original $\mathrm{PLMA}_{39}-\mathrm{PBzMA}_{294}$ spheres. This suggests that the hybridization mechanism involves initial expulsion of copolymer chains from the small $\mathrm{PLMA}_{39^{-}}$ PBzMA $_{97}$ spheres and their subsequent incorporation within the larger $\mathrm{PLMA}_{39}-\mathrm{PBzMA}_{294}$ spheres. Moreover, TEM and SAXS analyses indicate that weakly anisotropic nanoparticles can be obtained as a final (mixed) copolymer morphology over a restricted range of compositions (e.g. volume fractions of 0.20-0.35 for the smaller $\mathrm{PLMA}_{39}-\mathrm{PBzMA}_{97}$ spheres) when heating such binary mixtures of spheres up to $150{ }^{\circ} \mathrm{C}$. As far as we are aware, such non-isotropic species have not been observed for any nanoparticle hybridization experiments reported in the literature. Finally, we provide the first compelling experimental evidence for a micelle fusion-fission mechanism in such systems. A two-stage mechanism that involves both expulsion/insertion and micelle fusion/fission events is proposed to account for our observations.

\section{Conflicts of interest}

There are no conflicts to declare.

\section{Acknowledgements}

Lubrizol is thanked for funding a PhD studentship for E. J. C. and for permission to publish this work. S. P. A. acknowledges an EPSRC Established Career Particle Technology Fellowship (EP/R003009). We are grateful to the European Synchrotron Radiation Facility (ESRF, Grenoble, France) for providing synchrotron beam-time (SC-4384) and thank Dr R. Dattani and the personnel of ID02 for their assistance.

\section{References}

1 Y. Mai and A. Eisenberg, Chem. Soc. Rev., 2012, 41, 59695985.

2 C. J. Hawker and T. P. Russell, MRS Bull., 2005, 30, 952-966.

3 H. C. Kim, S. M. Park and W. D. Hinsberg, Chem. Rev., 2010, 110, 146-177.

4 W. J. Durand, G. Blachut, M. J. Maher, S. Sirard, S. Tein, M. C. Carlson, Y. Asano, S. X. Zhou, A. P. Lane, C. M. Bates, C. J. Ellison and C. G. Willson, J. Polym. Sci., Part A: Polym. Chem., 2015, 53, 344-352.

5 H. Yu, X. Qiu, N. Moreno, Z. Ma, V. M. Calo, S. P. Nunes and K.-V. Peinemann, Angew. Chem., Int. Ed., 2015, 54, 1393713941.

6 J. Lachaux, C. Alcaine, B. Gómez-Escoda, C. M. Perrault, D. O. Duplan, P. Y. J. Wu, I. Ochoa, L. Fernandez, O. Mercier, D. Coudreuse and E. Roy, Lab Chip, 2017, 17, 2581-2594.

7 R. Zheng, G. Liu, M. Devlin, K. Hux and T.-C. Jao, Tribol. Trans., 2010, 53, 97-107.

8 M. J. Derry, O. O. Mykhaylyk and S. P. Armes, Angew. Chem., Int. Ed., 2017, 56, 1746-1750.

9 I. Canton, N. J. Warren, A. Chahal, K. Amps, A. Wood, R. Weightman, E. Wang, H. Moore and S. P. Armes, ACS Cent. Sci., 2016, 2, 65-74.

10 W. Chen and J. Du, Sci. Rep., 2013, 3, 2162.

11 M. Szwarc, Nature, 1956, 178, 1168-1169.

12 G. Moad, E. Rizzardo and S. H. Thang, Aust. J. Chem., 2012, 65, 985-1076.

13 G. Moad, E. Rizzardo and S. H. Thang, Aust. J. Chem., 2009, 62, 1402-1472. 
14 G. Moad, E. Rizzardo and S. H. Thang, Polymer, 2008, 49, 1079-1131.

15 G. Moad, E. Rizzardo and S. H. Thang, Acc. Chem. Res., 2008, 41, 1133-1142.

16 S. Perrier, Macromolecules, 2017, 50, 7433-7447.

17 E. J. Cornel, S. Van Meurs, T. Smith, P. S. O'Hora and S. P. Armes, J. Am. Chem. Soc., 2018, 140, 12980-12988.

18 J. Chiefari, Y. K. Chong, F. Ercole, J. Krstina, J. Jeffery, T. P. T. Le, R. T. A. Mayadunne, G. F. Meijs, C. L. Moad, G. Moad, E. Rizzardo and S. H. Thang, Macromolecules, 1998, 31, 5559-5562.

19 G. Gody, T. Maschmeyer, P. B. Zetterlund and S. Perrier, Macromolecules, 2014, 47, 3451-3460.

20 E. E. Brotherton, F. L. Hatton, A. A. Cockram, M. J. Derry, A. Czajka, E. J. Cornel, P. D. Topham, O. O. Mykhaylyk and S. P. Armes, J. Am. Chem. Soc., 2019, 141, 13664-13675.

21 A. Blanazs, A. J. Ryan and S. P. Armes, Macromolecules, 2012, 45, 5099-5107.

22 L. A. Fielding, M. J. Derry, V. Ladmiral, J. Rosselgong, A. M. Rodrigues, L. P. D. Ratcliffe, S. Sugihara and S. P. Armes, Chem. Sci., 2013, 4, 2081-2087.

23 L. Houillot, C. Bui, C. Farcet, C. Moire, J.-A. Raust, H. Pasch, M. Save and B. Charleux, ACS Appl. Mater. Interfaces, 2010, 2, 434-442.

24 L. Houillot, C. Bui, M. Save, B. Charleux, C. Farcet, C. Moire, J.-A. Raust and I. Rodriguez, Macromolecules, 2007, 40, 65006509.

25 Y. Pei, O. R. Sugita, L. Thurairajah and A. B. Lowe, RSC Adv., 2015, 5, 17636-17646.

26 Y. Pei, J.-M. Noy, P. J. Roth and A. B. Lowe, J. Polym. Sci., Part A: Polym. Chem., 2015, 53, 2326-2335.

27 Y. W. Pei, A. B. Lowe and P. J. Roth, Macromol. Rapid Commun., 2017, 38, 1600528.

28 W. Zhao, G. Gody, S. Dong, P. B. Zetterlund and S. Perrier, Polym. Chem., 2014, 5, 6990-7003.

29 R. Lund, L. Willner, V. Pipich, I. Grillo, P. Lindner, J. Colmenero and D. Richter, Macromolecules, 2011, 44, 6145-6154.

30 T. Zinn, L. Willner, R. Lund, V. Pipich and D. Richter, Soft Matter, 2012, 8, 623-626.

31 T. Zinn, L. Willner, V. Pipich, D. Richter and R. Lund, ACS Macro Lett., 2016, 5, 884-888.

32 N. König, L. Willner, V. Pipich, T. Zinn and R. Lund, Phys. Rev. Lett., 2019, 122, 078001.

33 R. Lund, L. Willner, J. Stellbrink, P. Lindner and D. Richter, Phys. Rev. Lett., 2006, 96, 068302.

34 R. Lund, L. Willner, D. Richter and E. E. Dormidontova, Macromolecules, 2006, 39, 4566-4575.

35 T. Zinn, L. Willner, V. Pipich, D. Richter and R. Lund, ACS Macro Lett., 2015, 4, 651-655.

36 J. Lu, F. S. Bates and T. P. Lodge, Macromolecules, 2016, 49, 1405-1413.
37 J. Lu, F. S. Bates and T. P. Lodge, Macromolecules, 2015, 48, 2667-2676.

38 J. Lu, F. S. Bates and T. P. Lodge, ACS Macro Lett., 2013, 2, 451-455.

39 J. Lu, S. Choi, F. S. Bates and T. P. Lodge, ACS Macro Lett., 2012, 1, 982-985.

40 S.-H. Choi, F. S. Bates and T. P. Lodge, Macromolecules, 2011, 44, 3594-3604.

41 S. H. Choi, T. P. Lodge and F. S. Bates, Phys. Rev. Lett., 2010, 104, 47802 .

42 D. J. Growney, O. O. Mykhaylyk and S. P. Armes, Langmuir, 2014, 30, 6047-6056.

43 T. Nicolai, O. Colombani and C. Chassenieux, Soft Matter, 2010, 6, 3111.

44 E. E. Dormidontova, Macromolecules, 1999, 32, 7630-7644.

45 A. Halperin, Macromolecules, 2011, 44, 5072-5074.

46 A. Halperin and S. Alexander, Macromolecules, 1989, 22, 2403-2412.

47 T. Haliloğlu, I. Bahar, B. Erman and W. L. Mattice, Macromolecules, 1996, 29, 4764-4771.

48 Y. Wang, C. M. Kausch, M. Chun, R. P. Quirk and W. L. Mattice, Macromolecules, 1995, 28, 904-911.

49 Y. Ma and T. P. Lodge, Macromolecules, 2016, 49, 9542-9552. 50 A. S. Mansour, L. F. Johnson, T. P. Lodge and F. S. Bates, J. Polym. Sci., Part B: Polym. Phys., 2010, 48, 566-574.

51 M. J. Derry, L. A. Fielding and S. P. Armes, Polym. Chem., 2015, 6, 3054-3062.

52 A. P. Lopez-Oliva, N. J. Warren, A. Rajkumar, O. O. Mykhaylyk, M. J. Derry, K. E. B. Doncom, M. J. Rymaruk and S. P. Armes, Macromolecules, 2015, 48, 3547-3555.

53 L. A. Fielding, J. A. Lane, M. J. Derry, O. O. Mykhaylyk and S. P. Armes, J. Am. Chem. Soc., 2014, 136, 5790-5798.

54 J. S. Pedersen, J. Appl. Crystallogr., 2000, 33, 637-640.

55 J. S. Pedersen and M. C. Gerstenberg, Macromolecules, 1996, 29, 1363-1365.

56 P. Debye, J. Phys. Colloid Chem., 1947, 51, 18-32.

57 L. J. Fetters, D. J. Lohsey and H. Colby, Physical Properties of Polymers Handbook, Springer New York, 2nd edn, 2007.

58 D. Zhao, Y. Ma and T. P. Lodge, Macromolecules, 2018, 51, 2312-2320.

59 Neutrons, X-rays and Light: Scattering Methods Applied to Soft Condensed Matter, ed. P. Lindner and T. Zemb, Elsevier Netherlands, Amsterdam, 1st edn, 2002.

60 S. J. Byard, C. T. O'Brien, M. J. Derry, M. Williams, O. O. Mykhaylyk, A. Blanazs and S. P. Armes, Chem. Sci., 2020, 11, 396-402.

61 E. J. Cornel, G. N. Smith, S. E. Rogers, J. E. Hallett, D. J. Growney, T. Smith, P. S. O'Horah, S. van Meurs, O. O. Mykhaylyk and S. P. Armes, Soft Matter, 2020, 16, DOI: $10.1039 / \mathrm{c} 9 \mathrm{sm} 02425 \mathrm{e}$.

62 P. Chambon, A. Blanazs, G. Battaglia and S. P. Armes, Langmuir, 2012, 28, 1196-1205. 